Article

\title{
Technetium-Radiolabeled Mannose-Functionalized Gold Nanoparticles as Nanoprobes for Sentinel Lymph Node Detection
}

\author{
Oscar J. Estudiante-Mariquez ${ }^{1,2}$, Andrés Rodríguez-Galván ${ }^{3, *(\mathbb{D})}$, David Ramírez-Hernández ${ }^{1,2}$, \\ Flavio F. Contreras-Torres $4(\mathbb{D})$ and Luis A. Medina $1,5, *(\mathbb{D})$ \\ 1 Unidad de Investigación Biomédica en Cáncer INCan-UNAM, Instituto Nacional de Cancerología, Cd. \\ México 14080, Mexico; ojem96@gmail.com (O.J.E.-M.); 350davidramirez@gmail.com (D.R.-H.) \\ 2 Facultad de Química, Universidad Nacional Autónoma de México, Coyoacán, Cd. México 04510, Mexico \\ 3 Carrera de Biología, Unidad de Biomedicina, Facultad de Estudios Superiores Iztacala, Universidad \\ Nacional Autónoma de México, Tlalnepantla, Estado de México 54090, Mexico \\ 4 Escuela de Ingeniería y Ciencias, Tecnologico de Monterrey, Nuevo León 64849, Mexico; \\ contreras.flavio@itesm.mx \\ 5 Instituto de Física, Universidad Nacional Autónoma de México, Coyoacán, Cd. México 04510, Mexico \\ * Correspondence: andresrodriguezga@gmail.com (A.R.-G.); medina@fisica.unam.mx (L.A.M.); \\ Tel.: +52-55-3693-5200 (ext. 228) (A.R.-G.); +52-55-5622 5000 (ext. 2120) or \\ +52-55-3693-5200 (ext. 228) (L.A.M.)
}

Received: 6 March 2020; Accepted: 20 April 2020; Published: 23 April 2020

\begin{abstract}
Gold nanoparticles (AuNPs) are considered valuable nanomaterials for the design of radiolabeled nanoprobes for single-photon emission computed tomography (SPECT) imaging. Radiolabeled and functionalized AuNPs could improve lymphatic mapping by enhancing the radioactive signaling of individual particles in the sentinel node. In this study, an alternative method for functionalizing commercial AuNps with mannose is described. The chemical derivatization and biofunctionalization of AuNPs were performed with lipoic acid and mannose, respectively. Several levels of mannose were tested; the thiolate hydrazinonicotinamide-glycine-glycine-cysteine (HYNIC) molecule was also used for ${ }^{99} \mathrm{~m} \mathrm{Tc}$ radiolabeling. Physicochemical characterization of this system includes U-V spectroscopy, dynamic light scattering, Fourier-transform infrared spectroscopy, and transmission electron microscopy. The most stable nanoprobe, in terms of the aggregation, radiolabeling efficiency, and purity, was tested in a sentinel lymph node model in a rat by microSPECT/computed tomography (CT) imaging. The SPECT images revealed that ${ }^{99 \mathrm{~m}}$ Tc-radiolabeled AuNPs functionalized with mannose can track and accumulate in lymph nodes in a similar way to the commercial ${ }^{99} \mathrm{~m}$ Tc-Sulfur colloid, commonly used in clinical practice for sentinel lymph node detection. These promising results support the idea that ${ }^{99 \mathrm{~m}} \mathrm{Tc}-\mathrm{AuNPs}-\mathrm{mannose}$ could be used as a SPECT contrast agent for lymphatic mapping.
\end{abstract}

Keywords: gold nanoparticles; mannose; SPECT; sentinel lymph node; ${ }^{99 \mathrm{~m}} \mathrm{Tc}$

\section{Introduction}

Gold nanoparticles (AuNPs) have unique physical and chemical properties that make them valuable nanomaterials for many clinical applications, including their use as contrast media in diagnostic imaging [1]. The binding affinity of sulfur-based groups to gold surfaces facilitates the derivatization and functionalization of AuNPs with small molecules and biomolecules, allowing the manufacture of contrast agent systems with active targeting capabilities for multimodal imaging [2]. 
Functionalized AuNPs are ideal contrast agents for hybrid imaging techniques, for instance, single-photon emission computed tomography (SPECT) with computed tomography (CT) [3]. Radiolabeled AuNPs have been widely explored with SPECT imaging due to its high sensitivity, unlimited tissue penetration, and clinic translational capability. For example, ${ }^{125} \mathrm{I},{ }^{111} \mathrm{In},{ }^{131} \mathrm{I}$, and ${ }^{99} \mathrm{~m}$ Tc radionuclides have been attached to functionalized AuNPs [4-6], while the dual deposition of radionuclides (e.g., ${ }^{125} \mathrm{I}$ and ${ }^{111} \mathrm{In}$ ) has been used to prepare multimodal probes for SPECT bioimaging [7]. In particular, SPECT/CT mapping has demonstrated a high potential to improve preoperative sentinel lymph node (SLN) localization and the reduction of false negatives compared to either CT or ultrasound scans $[8,9]$. The SLN denotes the first node in the lymphatic chain draining a primary tumor and provides critical diagnostic and prognostic information for patients, since it can help to identify those with signs of metastasis. In fact, there is a growing interest in the development of new and improved SPECT contrast agents for SLN detection [10].

New contrast agents should improve SLN imaging by enhancing the radioactive signaling of individual particles in the tracking of the lymphatic net and/or increase the radiotracer delivery and retention in the sentinel node [10]. Functionalized nanoparticles are relevant in SLN imaging and targeting not only because these particles can be synthesized at well-defined sizes, but also because different radionuclides can be attached to their surface, which is prepared with ligands for the active targeting of structures located at the SLN. Precise localization of unclear lymphatic nodes requires radiolabeled contrast agents exhibiting size range variation between 20 and $50 \mathrm{~nm}$ to be easily removed from the injection site, transported within the lymphatic network, and retained for many hours in the nodes [11]. For the efficient imaging of lymphatic tracking, the ${ }^{99 \mathrm{~m}} \mathrm{Tc}$ is an ideal radionuclide because of its short 6-h half-life and photon energy of $140 \mathrm{keV}$, as well as its commercial availability and low cost $[11,12]$.

In a previous study, a multifunctional system of ${ }^{99 \mathrm{~m}} \mathrm{Tc}-$ labeled gold nanoparticles conjugated to HYNIC-GGC/mannose was prepared to evaluate its biological behavior as a potential radiopharmaceutical for sentinel lymph node detection in a rat model [13]. It was concluded that ${ }^{99 \mathrm{~m}} \mathrm{Tc}-\mathrm{AuNP}$-mannose remains within the first lymph node (the popliteal node) of Wistar rats for $24 \mathrm{~h}$, showing the potential of this radioconjugate for the specific targeting of SLN. In the current study, an alternative method for functionalizing commercial AuNPs with mannose (AuNPs-MAN) using lipoic acid is reported. This AuNPs-MAN system was also radiolabeled with ${ }^{99 \mathrm{~m}} \mathrm{Tc}$ to evaluate its potential for lymph node mapping using microSPECT/CT imaging in a rat model. Physicochemical characterization of this system includes U-V spectroscopy, dynamic light scattering, Fourier-transform infrared spectroscopy, and transmission electron microscopy.

\section{Results}

\subsection{Preparation and Characterization of ${ }^{99 m} T c-A u N P s-M A N$}

Table 1 shows the Z-potential value and hydrodynamic diameter of AuNP conjugates. The incubation of AuNPs at different times with lipoic acid (ALA) showed no significant changes. The conjugation with 2-aminoethyl 2,3,4,6-tetra-O-acetyl- $\alpha$-D-mannopyranoside hydrochloride (MAN) or hydrazinonicotinamide-glycine-glycine-cysteine (HYNIC) did not modify the Z-potential value. Previous studies have shown that AuNPs functionalized with carboxylic acids exhibited negative Z-potential values close to $-60 \mathrm{mV}$ [14], suggesting that ALA can be attached to gold nanoparticles via the dithiol ring, with the carboxylic acid extending out into solution. A successful covalent functionalization between $-\mathrm{NH}_{2}$ (MAN) and $-\mathrm{COOH}$ (ALA) moieties would lead to the formation of amide groups. 
Table 1. Z-potential values and hydrodynamic diameter (in parentheses) for synthesized particles. Values represent the mean value $\pm \mathrm{SD}$.

\begin{tabular}{ccccc}
\hline & $\mathbf{2} \mathbf{h}$ & $\mathbf{2 4} \mathbf{h}$ & $\mathbf{4 8} \mathbf{h}$ & $\mathbf{7 2} \mathbf{h}$ \\
\hline *uNPs & $(30 \pm 1.6)$ & $(33 \pm 6.5)$ & $(38 \pm 5.6)$ & $(31 \pm 4.1)$ \\
AuNPs-ALA & $-53.7 \pm 16.0$ & $-63.7 \pm 6.2$ & $-73.6 \pm 1.4$ & $-64.2 \pm 4.1$ \\
& $(73 . \pm 31.3)$ & $(73 . \pm 36.3)$ & $(60.3 \pm 23.2)$ & $(59.9 \pm 23.9)$ \\
AuNPs-MAN & $-58.5 \pm 8.1$ & $-65.9 \pm 7.4$ & $-67.8 \pm 10.4$ & $-60.25 \pm 8.38$ \\
& $(103.5 \pm 46.4)$ & $(82.1 \pm 37.9)$ & $(71.1 \pm 18.7)$ & $(69.8 \pm 19.3)$ \\
HYNIC-AuNPs-MAN & $-59.2 \pm 10.8$ & $-59.5 \pm 6.5$ & $-59.7 \pm 7.2$ & $-71.1 \pm 10.6$ \\
& $(72.6 \pm 51.6)$ & $(76.8 \pm 28.7)$ & $(49.8 \pm 14)$. & $(68.6 \pm 29.4)$ \\
\hline
\end{tabular}

* Z-potential is presented in $\mathrm{mV}$ and size is presented in $\mathrm{nm}$. All measurements were conducted in deionized water at $\mathrm{pH} 7.0$ adjusted with $\mathrm{NaOH}(1 \mathrm{M})$.

Increments in the hydrodynamic diameter were also not statistically significant; in fact, the Polydispersity Index was greater than 0.2 in all groups, indicating the presence of different size populations. It is well-known that the Dynamic Light Scattering (DLS) method gives an estimation of the hydrodynamic diameter of the particle, but it can be greatly affected by the particle shape and structure. In addition, a small portion of large contaminant or aggregated particles can greatly contribute to scattering; it is possible that the inclusion of ALA, MAN, and HYNIC attached to the surface of AuNps affects the estimation of the hydrodynamic diameter, giving information on the AuNp core, along with the coating and the layer of solvent particles attached to its surface [15]. These observations are consistent with the amount of ALA molecules chemisorbed on the surface of AuNP nanoparticles.

Figure 1 illustrates the attenuated total reflection Fourier transform infrared (FTIR-ATR) studies for the covalent bonding of MAN molecules on ALA moieties. The AuNPs-ALA 48 spectra (red line) show a peak at $1700 \mathrm{~cm}^{-1}$ that was assigned to $\mathrm{C}=\mathrm{O}$ double bond stretching. The lipid hydrocarbon chains contribute peaks at about $2848 \mathrm{~cm}^{-1}\left(\mathrm{CH}_{2}\right.$, symmetric stretching) and $2920 \mathrm{~cm}^{-1}\left(\mathrm{CH}_{2}\right.$, asymmetric stretching). After the covalent bond (gray line), AuNPs-MAN ${ }_{48}$ shows a peak at $\sim 3400$ that was assigned to O-H stretching. The peak at $2970 \mathrm{~cm}^{-1}$ was assigned to $\mathrm{C}-\mathrm{H}$ stretching of the pyranosic sugar rings, while the peak at $1650 \mathrm{~cm}^{-1}$ was assigned to the stretching band of $\mathrm{C}=\mathrm{O}$ stretching and C-N stretching. Furthermore, the $1580 \mathrm{~cm}^{-1}$ peak was associated with in-plane N-H bending and C-N stretching, while the peak at $1280 \mathrm{~cm}^{-1}$ was related to C-N stretching. Finally, the peak at $1070 \mathrm{~cm}^{-1}$ was related to C-O stretching [16].

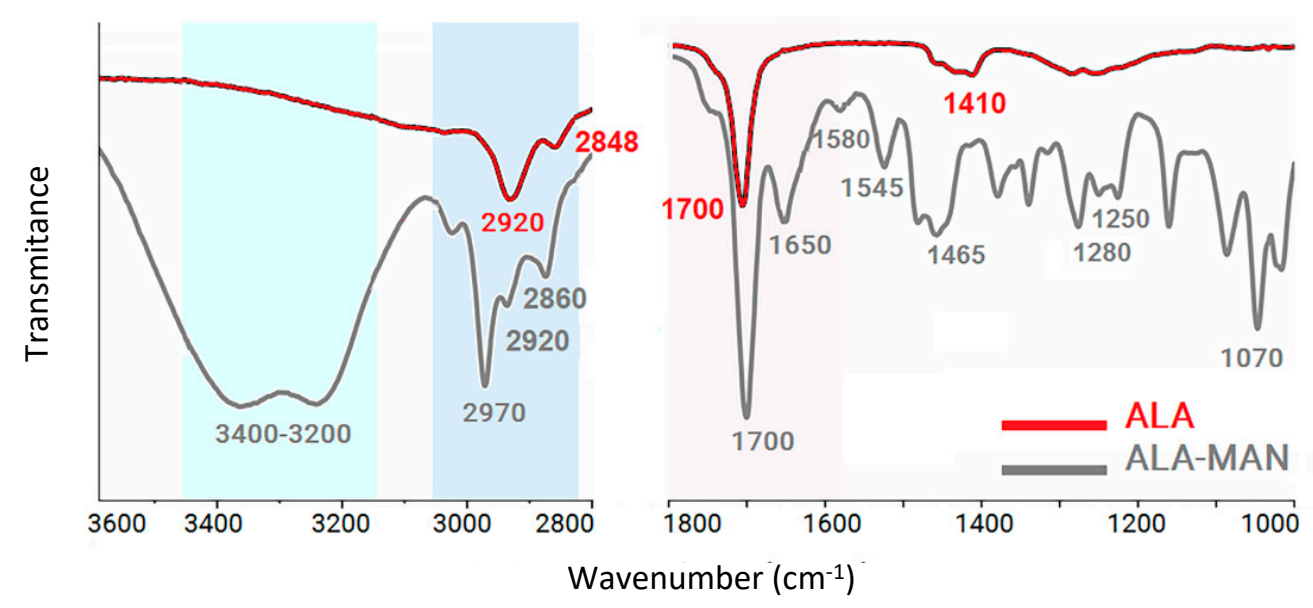

Figure 1. The attenuated total reflection Fourier transform infrared (ATR-FTIR) spectra for the covalent bonding of 2-aminoethyl 2,3,4,6-tetra-O-acetyl- $\alpha$-D-mannopyranoside hydrochloride (MAN) molecules on lipoic acid (ALA) moieties. 
The UV-Vis spectroscopy analysis of AuNPs-ALA, AuNPs-MAN, and HYNIC-AuNPs-MAN conjugates is presented in Figure 2. The AuNPs have an extinction maximum peak $\left(\lambda_{\max }\right)$ at about 521 $\mathrm{nm}$. Although the localized surface plasmon resonance (LSPR) spectrum is very sensitive to changes in the nanoparticle's shape, size, stability, surrounding medium, and/or surface modifications [14], the location of the maximum peak does not change significantly after conjugation with ALA (Figure 2a). However, some broadening was observed for the 2 and $24 \mathrm{~h}$ conjugates after the covalent functionalization with MAN (Figure 2b). After conjugation with HYNIC, only the 2 h conjugate maintained the shift broadening (Figure 2c). The UV-Vis analysis indicates that in terms of aggregation, the conjugate with $48 \mathrm{~h}$ incubation is the most stable system.
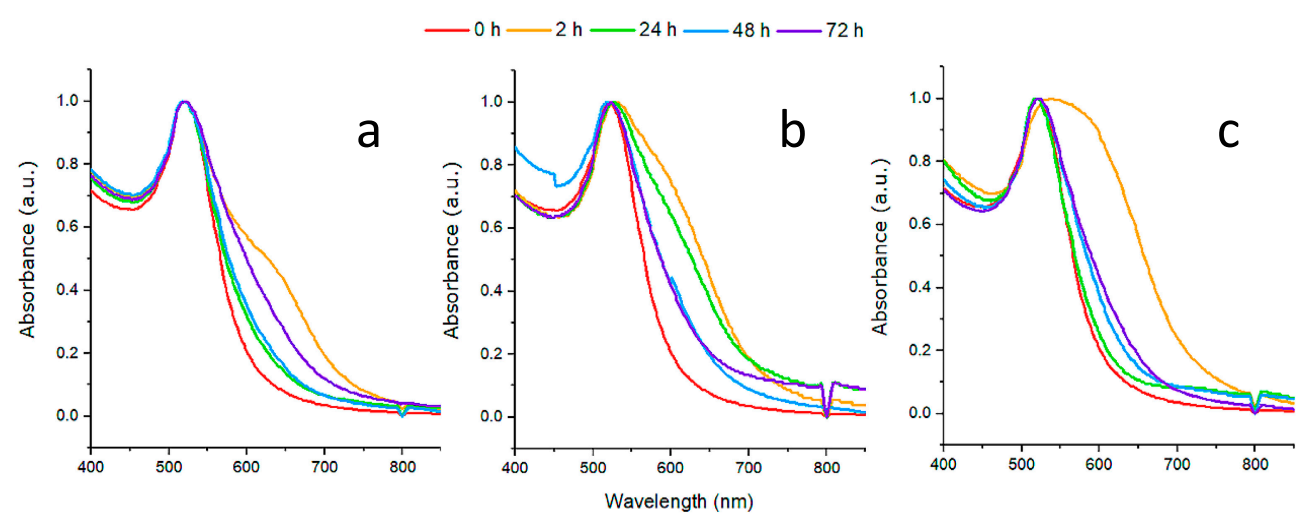

Figure 2. Ultraviolet-visible spectra for: (a) Gold nanoparticles (AuNPs)-ALA, (b) AuNPs-MAN, and (c) hydrazinonicotinamide-glycine-glycine-cysteine (HYNIC)-AuNPs-MAN. The red color indicates the spectra of pristine gold nanoparticles and the yellow, green, blue, and purple colors indicate the incubation times at $2,24,48$, and $72 \mathrm{~h}$, respectively.

Figure 3 shows the transmission electron microscopy images and HRTEM analysis. It was observed that the conjugates were spherical and no evident changes in the average particle size occurred (see the histograms of the size distribution). The average size of pristine AuNPs was $18.57 \pm 2.55 \mathrm{~nm}$ and the size after functionalization with ALA and HYNIC was $18.95 \pm 2.54$ and $18.87 \pm 2.37$, respectively; no statistical differences were observed. A halo of low electron density material was observed around the functionalized gold nanoparticle, which was related to the presence of an ALA or MAN coating. The HRTEM elemental analysis confirmed the metallic nature of nanoparticles, as well as the presence of $\mathrm{N}$ and $\mathrm{S}$ elements in the organic molecules related to ALA and MAN. 

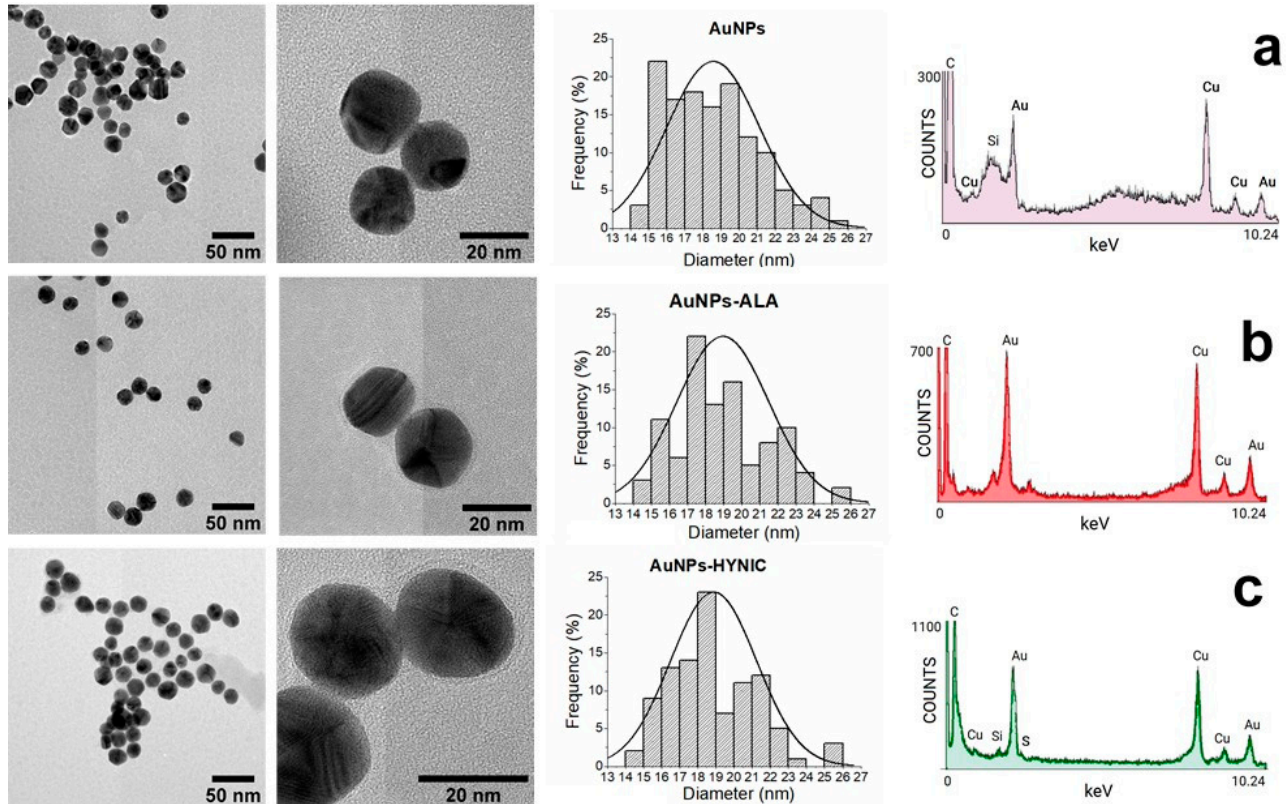

Figure 3. Representative transmission electron microscopy images, size histogram distribution, and HRTEM analysis for (a) AuNPs, (b) AuNPs-ALA, and (c) AuNPs-MAN. Results correspond to the $48 \mathrm{~h}$ incubation group.

\subsection{Radiolabeling Analysis}

According to the DLS, Z-potential and UV-Vis results, the AuNPs-MAN ${ }_{48 \mathrm{~h}}$ and AuNPs-MAN $72 \mathrm{~h}$ conjugates were selected for ${ }^{99 \mathrm{~m}} \mathrm{Tc}$ radiolabeling. Table 2 shows the results for the radiolabeling efficiency and stability, and the radiochemical purity. The radiochemical purity is important in radiopharmacy and SPECT imaging since it is the radiochemical form that determines the pharmacokinetics and biodistribution of the radiopharmaceutical. Given these results, AuNPs-MAN $48 \mathrm{~h}$ was used for the in vivo SPECT/CT imaging study.

Table 2. Radiolabeling results.

\begin{tabular}{cccc}
\hline Conjugate & $\begin{array}{c}\text { Radiolabeling } \\
\text { Efficiency }\end{array}$ & Radiochemical Purity & Radiolabeling Stability * \\
\hline${ }^{99 m}$ Tc-AuNPs-MAN $48 \mathrm{~h}$ & $86 \pm 14 \%$ & $97 \pm 2 \%$ & $99 \pm 2.3 \%$ \\
${ }^{99 m}$ Tc-AuNPs-MAN $72 \mathrm{~h}$ & $60 \pm 30 \%$ & $84 \pm 20 \%$ & $96 \pm 2.1 \%$ \\
\hline
\end{tabular}

* In vitro stability assay in saline solution $(\mathrm{NaCl} 0.9 \%)$ and fresh human serum for a $6 \mathrm{~h}$ period.

\subsection{In Vivo SPECT/CT Imaging}

Figure 4 depicts the procedure used to evaluate the lymphatic mapping of the ${ }^{99 \mathrm{~m}} \mathrm{Tc}-\mathrm{AuNPs}-\mathrm{MAN}_{48 \mathrm{~h}}$. After an injection in the footpad, the contrast agent drained from the administration site (AA) into the first lymphatic node, i.e., the popliteal lymph node (PO). Next, the contrast followed the lymphatic drainage toward the iliac lymph node (IL) and then the renal node (RE). During the injection of the contrast agents, it was noticed that the ${ }^{99 \mathrm{~m}} \mathrm{Tc}-\mathrm{Sulfur}$ colloid induced a significant irritation (observed as a pain reaction in the footpad during and after inoculation); this situation was not observed with the conjugated gold nanoparticles. 


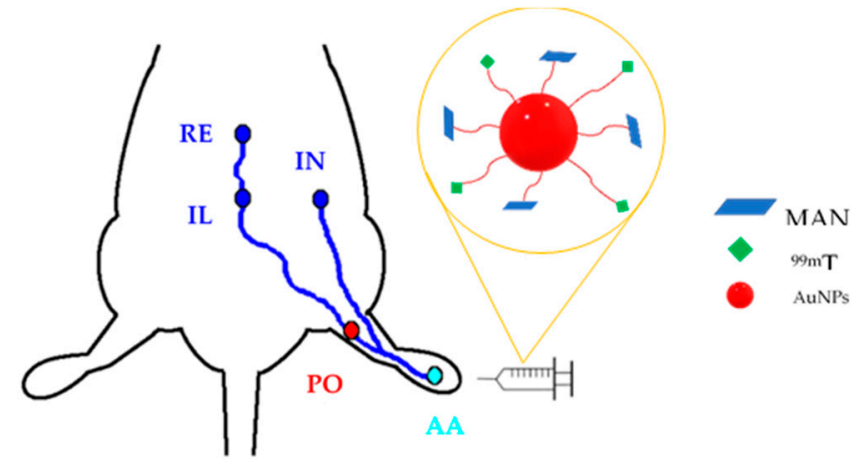

Figure 4. Illustrative picture of the ${ }^{99 \mathrm{~m}} \mathrm{Tc}-\mathrm{AuNPs}_{\mathrm{N}} \mathrm{MAN}_{48 \mathrm{~h}}$ mapping in lymphatic vessels and nodes in a rat. The lymph nodes are identified as popliteal (PO), iliac (IL), inguinal (IN), and renal (RE).

Figure 5 shows representative 3D microSPECT/CT images for Group A. Here, ${ }^{99} \mathrm{~m}$ Tc-AuNPs-MAN $48 \mathrm{~h}$ was injected in the right footpad, while ${ }^{99 \mathrm{~m}} \mathrm{Tc}-\mathrm{AuNPs}-\mathrm{ALA}_{48 \mathrm{~h}}$ was injected in the left one. Lead covers were placed at the footpads to block the signal from the injection site. The images depict a preferential accumulation of AuNPs-MAN ${ }_{48 \mathrm{~h}}$ in the PO node $3 \mathrm{~h}$ post injection. A minimal accumulation of AuNPs-ALA $48 \mathrm{~h}$ was observed in the left PO node, except for the third rat (R3 in the figure). In this rat, accumulation in the IL and right IN nodes was also noticed. These results demonstrate mannose's ability to promote gold nanoparticle retention within the lymph nodes.

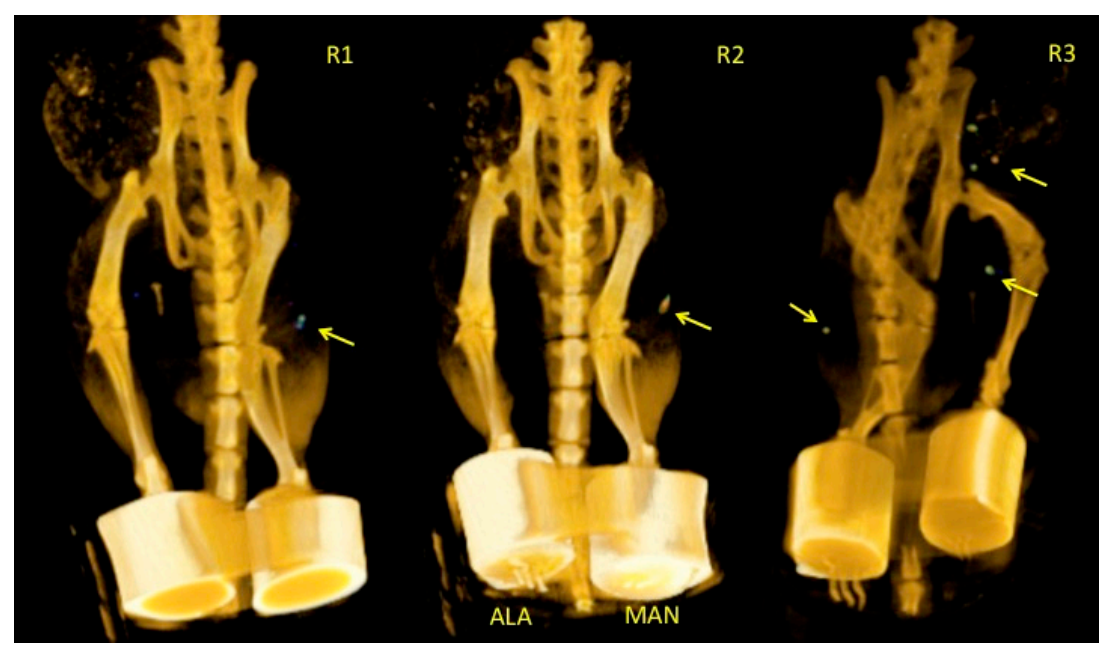

Figure 5. Micro single-photon emission computed tomography (SPECT)/computed tomography (CT) images depicting AuNPs-MAN $48 \mathrm{~h}$ vs. AuNPs-ALA $48 \mathrm{~h}$ accumulation in the popliteal lymph node. Arrows indicate the location of the nodes. ALA and MAN notation indicates the injection of AuNPs-ALA $48 \mathrm{~h}$ and AuNPs-MAN $48 \mathrm{~h}$ in the left and right footpad, respectively. Animals were oriented in a prone position.

Table 3 depicts the average percentage of the injected dose of AuNPs-MAN $48 \mathrm{~h}$ and AuNPs-ALA $48 \mathrm{~h}$ that stays at the injection site and the popliteal nodes. Values were calculated from the in vivo images and the ex vivo radioactivity quantification after animal sacrifice. Differences between in vivo and ex vivo results were not significant. 
Table 3. Average percentage of injected dose at injection site and popliteal nodes.

\begin{tabular}{ccccc}
\hline & \multicolumn{2}{c}{ AuNPs-ALA $_{\text {48h }}$} & \multicolumn{2}{c}{ AuNPs-MAN } \\
& Injection Site & Popliteal Node & Injection Site & Popliteal Node \\
\hline In vivo & $74.2 \pm 9.4$ & $1.0 \pm 0.3$ & $79.3 \pm 11.5$ & $3.1 \pm 2.7$ \\
Ex vivo & $80.4 \pm 6.9$ & $3.4 \pm 1.3$ & $77.4 \pm 5.3$ & $3.1 \pm 0.9$ \\
\hline
\end{tabular}

Values represent the average $\pm S D$ from $n=3$. Values were corrected by radioactive decay.

Figure 6 shows representative 3D microSPECT/CT images from Group B, illustrating the lymphatic mapping sequence in two different rats at 0,3 , and $6 \mathrm{~h}$ after injection of ${ }^{99 \mathrm{~m}} \mathrm{Tc}-\mathrm{Sulfur}$ colloid (left footpad) and ${ }^{99 m}$ Tc-AuNPs-MAN 48 h (right footpad). The accumulation of both contrast agents in the popliteal nodes could be observed from the beginning of the study; both contrast agents were retained in the popliteal nodes in different proportions and some moved to the next lymph nodes. Similar behavior was observed in the rest of the rats. This temporal retention of the contrast agent in the lymph nodes means that it can be employed in "radioactive staining" in order to improve SLN detection and identification.

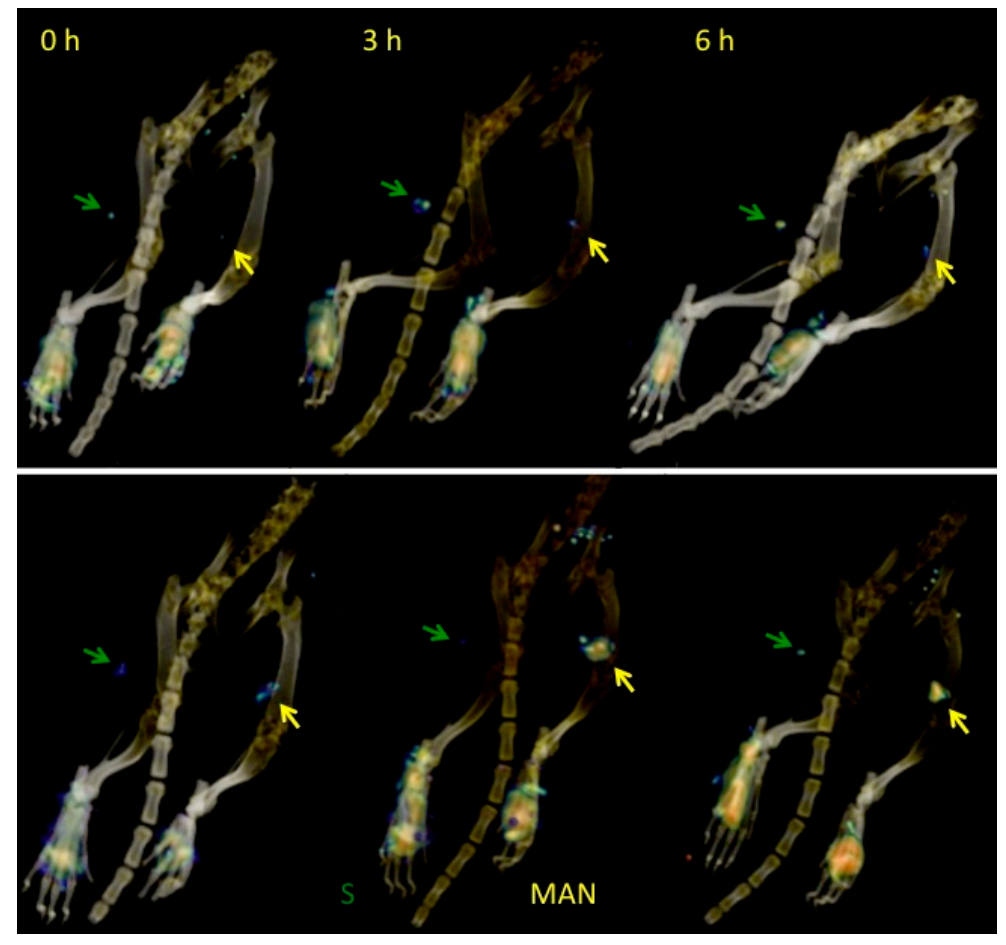

Figure 6. MicroSPECT/CT images of two different rats at different times, illustrating the differences in

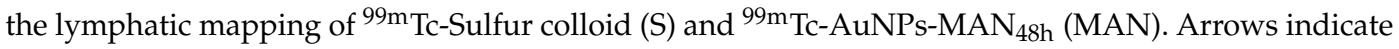
the location of the lymph nodes. Animals were oriented in a prone position.

A quantitative image analysis is presented in Figure 7; this figure describes variations as a function of time in terms of the percentage of injected dose at the injection site and popliteal lymph node. A box and whisker plot was used to illustrate the variability observed in the lymphatic mapping of each rat after the injection of both contrast media. The analysis indicates that the median and mean are almost equal. Certainly, the sample size is small, with $n=5$ (one rat was not used because the images showed a dissimilar distribution of the contrast agents, related to a bad injection procedure), but it exposes the tendency of data. No statistical differences were observed, indicating a similar behavior in the lymphatic mapping and node targeting of both contrast media. The results indicate that both contrast media moved to the next nodes (the IL and IN nodes (Figure 5)). However, the percentage of 
injected dose accumulated in the popliteal node (median values of 3.1-4.3 and 4.5-4.7, at 3 and $6 \mathrm{~h}$, respectively) allowed their detection and identification.
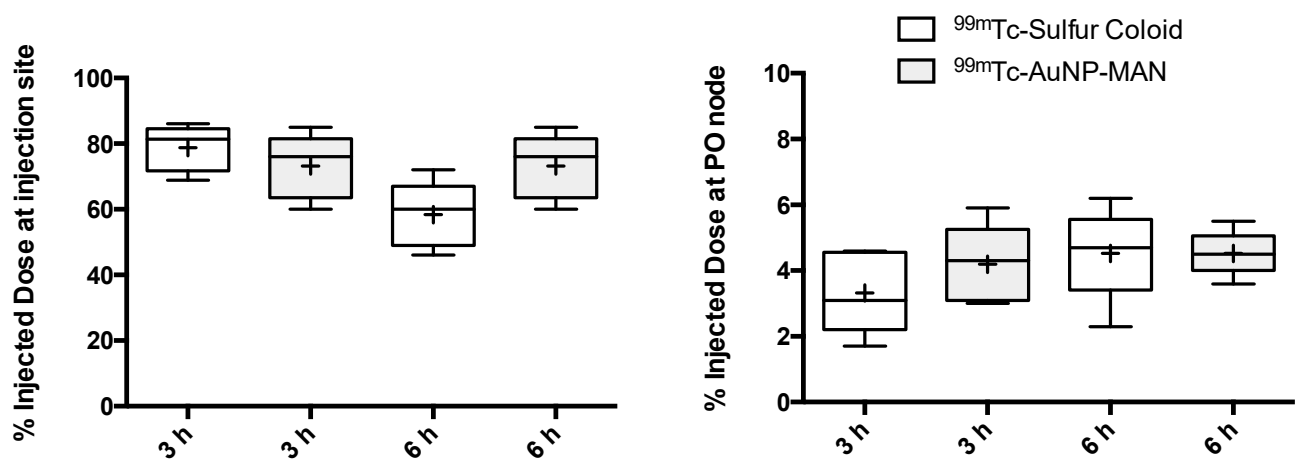

Figure 7. A box and whisker plot showing the results of the percentage of injected dose at the injection site (left) and popliteal node (right) at 3 and $6 \mathrm{~h}$ after the injection of either ${ }^{99 \mathrm{~m}} \mathrm{Tc}-\mathrm{Sulfur}$ colloid or ${ }^{99 m}$ Tc-AuNPs-MAN $48 \mathrm{~h}$. Boxes extend from 1st to 3rd quartiles; thick lines and + symbols inside boxes represent the median and mean $(n=5)$, respectively; whiskers go from the smallest to the largest values in each group and there were no outliers. No statistical difference was observed after ANOVA.

\section{Discussion}

There is current interest in developing new contrast media for sentinel lymph node mapping $[17,18]$. In cancer therapy, the lymph node status is a good indicator of the aggressiveness of neoplasm diseases, being the factor with the greatest prognostic importance and of fundamental consideration in treatment [19]. The sentinel lymph node (SLN) is the closest node that receives lymph from the primary tumor and is the most likely place for individual cancer cells or micrometastases to be located. The presence of cancer cells in the lymph node (or nodes) is interpreted as the presence of metastasis in the patient [18]. SLN biopsy is a highly reliable method for determining the stage of the tumor, and it also helps to calculate the risk of tumor cells spreading through the lymphatic system and to plan the most appropriate treatment (radiotherapy and/or dissection). Current SLN mapping methods are intraoperative; they need to use blue dye and/or a nanometer-sized radioactive colloid injection in order to perform a visual and radioactive detection of SLN. This method is limited by the rapid movement of blue dye and the low spatial resolution and sensitivity of radiation counters in detecting radioactive colloids [20]. One of the great advantages of the timely detection of SLN is that it can help prevent more extensive lymph node surgeries. Desirable characteristics for the new contrast media includes specificity towards molecular or cellular components that characterize the lymph nodes, multifunctionality to be able to add components for imaging and/or therapy, and a cost that competes with the contrast media currently used in the clinic $[6,10,13]$.

In the current work, we have evaluated a simple method for functionalizing commercial AuNps $(\sim 20 \mathrm{~nm})$ with mannose in order to be used in SLN detection. The HRTEM, spectroscopy, and DLS techniques implemented in this study have demonstrated that AuNPs can be successfully functionalized with ALA, MAN, and HYNIC. The TEM results have shown that the average core size of gold nanoparticles does not change throughout the functionalization process (Figure 3); DLS sizing of AuNPs-MAN $\mathrm{M}_{48}$ results in hydrodynamic diameters (i.e., the size that indicates how the particle behaves in a fluid) in the rage of 50-70 $\mathrm{nm}$ (Table 1). These sizes are similar in range to the current ${ }^{99} \mathrm{~m}$ Tc-labeled colloids employed for SLN detection: Nanocoll (albumin colloid, size ranging from 80 to $200 \mathrm{~nm}$.), Nanocis (colloidal rhenium sulphide, size range of 8-68 nm); and Hepatate (tin colloid, size range of 33-255 nm) [21]. The most stable nanoprobes, in terms of their physicochemical characteristics and radiochemical's purity and stability, were further radiolabeled with ${ }^{99 \mathrm{~m}} \mathrm{Tc}$ and successfully tested for lymph node mapping by microSPECT/CT in a rat model (Figures 5 and 6). 
To date, there are no commercial contrast agents with an optimal particle range size; however, there is a consensus that the contrast should be small enough to be rapidly removed from the injection site and transported to lymphatic nodes, and yet large enough to be retained for many hours in lymph nodes [22]. Small particles of less than $20 \mathrm{~nm}$ are usually cleared from the injection site and exchanged through blood capillaries. Particles of around 50 and $200 \mathrm{~nm}$ travel across the lymphatic capillaries and are trapped in the first lymph node [8]; large particles of hundreds of nanometers are trapped in the interstitial space and can be retained for long periods. Therefore, the hydrodynamic size of the AuNPs-MAN 48 nanoprobes is the standard size of commercial colloids used in SLN detection.

As was shown with the SPECT/CT studies, the conjugate ${ }^{99 m}$ Tc-AuNPs-MAN 48 stained the popliteal lymph node (i.e., the first draining lymph node in our experimental model) in a similar way to commercial ${ }^{99 \mathrm{~m}}$ Tc-Sulfur colloid, which is commonly used in clinical practice. This result partially supports the potential use of ${ }^{99 m}$ Tc-AuNPs-MAN 48 in lymphatic mapping. A significant difference observed between both agents was the minimal irritation induced by AuNPs-MAN 48 at the injection site compared with the sulfur colloid, which is a common side effect reported in the clinic. Another advantage of mannose-functionalized AuNPs is their potential use for multimodal photoacoustic SLN mapping, where binding to specific macrophage receptors is needed in order improve the specificity of the method $[23,24]$. Here, the use of mannose molecules for active targeting of the lymph node could increase the high affinity of the macrophage mannose receptor (MR, CD206), which is a C-type lectin predominantly expressed by most tissue macrophages, dendritic cells, and specific lymphatic or endothelial cells [25]. This receptor is found in lymphoid tissue at a high density and recognizes and binds to the mannose's carbohydrate side chains [2,3]. Because the AuNPs-MAN contains a high number of mannose molecules, it shows a multivalence effect that promotes a high binding affinity (avidity) for the mannose receptor [4,26].

Finally, a slow rate of movement from the injection site was noticed for both contrast agents (Figure 3). It is important to mention that the rat's footpads were massaged for a short period of time $(1 \mathrm{~min})$ after the injection, resulting in a poor stimulation for promoting the draining of the agents into the lymphatic track. It is possible that longer massaging times could result in better draining from the injection site, promoting fast accumulation of the contrast agents in the lymph nodes. However, slow draining could also permit the injection site to become a contrast depot, allowing continuous irrigation into the lymph nodes for a longer period of time.

\section{Conclusions}

In this study, we have reported a simple but efficient method for elaborating a radioactive contrast agent ( ${ }^{99 \mathrm{~m}}$ Tc-AuNPs-MAN) with a high potential for lymphatic mapping using SPECT/CT imaging. In vivo studies have shown that this system can track and accumulate in lymph nodes in a similar way to the commercial ${ }^{99 \mathrm{~m}}$ Tc-Sulfur colloid, which is commonly used in clinical practice for SLN detection. These results support the promising use of ${ }^{99 m}$ Tc-AuNPs-MAN in SLN detection; however, more studies are required to evaluate the AuNPs-MAN's toxicity, biodistribution, and pharmacokinetics before validating its potential clinical use for sentinel lymph node detection and targeting in cancer treatment.

\section{Materials and Methods}

\subsection{Materials}

Gold nanoparticles (AuNPs) with a $20 \mathrm{~nm}$ diameter (stabilized suspension in citrate buffer); lipoic acid (ALA), N-(3-dimethylaminopropyl)- $N^{\prime}$-ethylcarbodiimide hydrochloride (EDC), sodium hydroxide $(\mathrm{NaOH})$, ethylenediamine- $N, N^{\prime}$-diacetic acid (EDDA), tricine, tin(II) chloride $\left(\mathrm{SnCl}_{2}\right)$, and hydrochloric acid $(\mathrm{HCl})$ were purchased from Sigma-Aldrich (Mexico City, Mexico). 2-aminoethyl 2,3,4,6-tetra-O-acetyl- $\alpha$-D-mannopyranoside hydrochloride (MAN) was purchased from Synthose Inc, (Ontario, Canada). The modified peptide hydrazinonicotinamide-glycine-glycine-cysteine (HYNIC) was acquired from Peptides International (Kentucky, US). The ${ }^{99 \mathrm{~m}}$ Tc pertechnetate and ${ }^{99 \mathrm{~m}} \mathrm{Tc}-\mathrm{Sulfur}$ 
colloid were purchased from Instituto Nacional de Investigaciones Nucleares (Mexico, Mexico). All reagents were used as received, without further purification. Deionized water $(18.2 \mathrm{M} \Omega \mathrm{cm})$ was used in the experiments.

\subsection{Preparation of ${ }^{99 m} T c-A u N P s-M A N$}

\subsubsection{AuNPs-ALA}

Pristine AuNPs (3 mL, $1 \mathrm{nM})$ in sodium citrate were washed three times $(11,500 \mathrm{rpm}, 30 \mathrm{~min})$ and resuspended in deionized water at $\mathrm{pH} 11(\mathrm{NaOH}, 1 \mathrm{M})$. Then, $2.5 \mathrm{~mL}$ of AuNPs $(1 \mathrm{nM})$ was incubated with $250 \mu \mathrm{L}$ of ALA $(10 \mathrm{mM})$ for 2, 24, 48, or $72 \mathrm{~h}$ at room temperature and constant stirring of 3000 rpm [25]. For each time of incubation, free ALA was removed by ultrafiltration (Ultrafree-PF filters 10,000 NWWL, Millipore); the AuNPs-ALA pellets were washed three times (11,500 rpm, $30 \mathrm{~min})$ and resuspended in deionized water at $\mathrm{pH} 7$ and adjusted to a nanoparticle concentration of $1 \mathrm{nM}$ using Equation (1) [27]:

$$
c=A_{450} / \varepsilon_{450}
$$

where $A_{450}$ is the absorption of gold nanoparticles at $450 \mathrm{~nm}$ for a standard path length $l$ of $1 \mathrm{~cm}$ and $\varepsilon_{450}$ is the molar decadic extinction coefficient at $\lambda=450 \mathrm{~nm}$ for $20 \mathrm{~nm}$ gold nanoparticles. These conjugates were labeled according the incubation time, AuNPs-ALA $(\mathrm{h})$, (where, $\mathrm{h}=2,24,48$, or $72 \mathrm{~h}$ ).

The number of ALA molecules bonded to AuNPs was estimated by a UV-vis calibration curve of ALA concentrations (from 0.5 to $1.5 \mathrm{mM}$, plus a blank sample). The specific absorption peak at $330 \mathrm{~nm}$ of the five-membered ring in lipoic acid [28] was selected for the quantification. A UV-vis spectrophotometer (Beckman Coulter, California, US) was used to obtain the absorption spectrum as a function of the number of ALA molecules at each molar concentration. From a linear fit of data, the number of remaining free ALA molecules in solution after centrifugation was calculated; these values were used to estimate the number of ALA molecules per AuNp: 1107 \pm 587 at 2 h, $4151 \pm 4381$ at $24 \mathrm{~h}, 4428 \pm 479$ at $48 \mathrm{~h}$, and $5535 \pm 2089$ at $72 \mathrm{~h}$. The number of nanoparticles was calculated using equation (2):

$$
N=M_{C / m}
$$

where $M_{C}$ is the mass concentration of gold $(\mathrm{g} / \mathrm{mL})$ and $m$ is the mass of an individual nanoparticle (g/particle).

\subsubsection{AuNPs-MAN}

One milliliter of each AuNPs-ALA $(\mathrm{h})(1 \mathrm{nM}, \mathrm{pH} 7)$ was mixed with $2 \mu \mathrm{L}$ of MAN $(100 \mathrm{mg} / \mathrm{mL}$ in ethanol) and $10 \mu \mathrm{L}$ of EDC (40 mM, pH 6.5). These conjugates were labeled as AuNPs-MAN $(\mathrm{h})(\mathrm{h}=2$, $24,48$, or $72 \mathrm{~h}$ ). The solutions were incubated with constant stirring (3000 rpm, $5 \mathrm{~h})$, centrifuged at 11,500 rpm (30 min), washed three times in deionized water at $\mathrm{pH} 11$, and left in basic hydrolysis at $\mathrm{pH}$ 11 for $24 \mathrm{~h}$; finally, AuNPs-MAN $(\mathrm{h})$ conjugates were adjusted to a nanoparticle concentration of $1 \mathrm{nM}$.

\subsubsection{HYNIC-AuNPs-MAN}

One milliliter of each AuNPs-MAN $(\mathrm{h})(1 \mathrm{nM})$ was incubated with HYNIC $(5 \mu \mathrm{L}, 1 \mathrm{mM}$ in ethanol) with constant stirring (3000 rpm, $20 \mathrm{~min})$. The conjugates were labeled as HYNIC-AuNPs-MAN $(\mathrm{h})$ ( $\mathrm{h}=2,24,48$, or $72 \mathrm{~h}$ ). Unbound HYNIC was removed by ultrafiltration (Ultrafree-PF filters 10,000 NWWL, Millipore). The nanoparticles were washed three times (11,500 rpm, $30 \mathrm{~min})$, resuspended in deionized water at $\mathrm{pH} 11$, and adjusted to a nanoparticle concentration of $1 \mathrm{nM}$.

The number of HYNIC molecules per AuNP was also estimated by a UV-vis calibration curve from HYNIC (from 2 to $7 \mu \mathrm{M}$, plus a blank sample). The absorbance peak at $254 \mathrm{~nm}$ was used [29]; the absorption spectrum was plotted as a function of the number of HYNIC molecules at each molar concentration. The parameters from the linear fit of data were used to calculate the remaining free HYNIC molecules in solution after centrifugation; then, the number of HYNIC molecules per 
nanoparticle was estimated as $1199 \pm 430$ at $20 \mathrm{~min}, 2214 \pm 146$ at 2 h, $3063 \pm 360$ at 24 h, and $2989 \pm$ 387 at $48 \mathrm{~h}$. The number of nanoparticles was calculated using Equation (2).

\subsubsection{Characterization}

Absorption spectra (400-1000 nm) for each AuNPs-MAN $(\mathrm{h})$ were obtained with a Beckman Coulter DU-530 Life science UV/Vis spectrophotometer using a $1 \mathrm{~cm}$ quartz cuvette. UV/Vis analysis was used to monitor the AuNP surface plasmon band $(520 \mathrm{~nm})$ shift in order to evaluate the conjugation stability.

The hydrodynamic diameter and Z-potential were measured by Dynamic Light Scattering (DLS) and Electrophoretic Light Scattering, respectively, using a Z-sizer 90Plus Analyzer (Brookhaven Instruments Corporation, Long Island, NY, USA). In this equipment, the default calculation employs the Smoluchowski limit to calculate the Z-potential and the Stokes-Einstein equation for particle sizing. Smoluchowski approximation is valid for nanoparticles in aqueous media, even though the smallest nanoparticles ( $<20 \mathrm{~nm}$ diameter) may not have $\mathrm{ka}>>1$ necessary to justify the application of Smoluchowski approximation [30]. $\mathrm{ka}>>1$ indicates that the particle radius (a) is large compared to the Debye length $(1 / \mathrm{k})(1 / \mathrm{k}$ is $\sim 10 \mathrm{~nm}$ for $1 \mathrm{mM}$ aqueous salt solutions), which is used in Henry's function $(\mathrm{F}(\mathrm{ka}))$ to calculate the electrophoretic mobility using Henry's equation [30]. In this work, samples ( 20 nm AuNps) were analyzed in deionized water and adjusted to pH 7 with $1 \mathrm{M} \mathrm{NaOH}$ at $25^{\circ} \mathrm{C}$, and the final concentration was $1 \mathrm{mM}$ of $\mathrm{NaOH}$. Polystyrene nanospheres (100 nm) (Duke Scientific, California, US) and BI-ZR3 zeta potential reference material (Brookhaven Instruments Corp., New York, US) were used as standards to verify the equipment performance before measurements.

The attenuated total reflection Fourier transform infrared (FTIR-ATR) spectra, from 1000 to $3800 \mathrm{~cm}^{-1}$, of the covalent reaction for ALA-MAN, were recorded on a Perkin-Elmer Spectrum 100 spectrometer (Perkin Elmer, Ohio, US) to corroborate the formation of amide I and II bonds by comparing the spectra of AuNPs-ALA 48 before and after its conjugation with MAN. Samples were deposited on the ATR crystal by the drop casting technique and deposition was repeated several times.

Morphology and chemical composition analysis were performed using a JEM-2010F FASTEM instrument (JEOL, Massachusetts, US) coupled to a NORAN energy dispersive spectrophotometer (EDS) operating at $20 \mathrm{kV}$. Samples were prepared by applying $30 \mu \mathrm{L}$ of a diluted nanoparticle solution onto carbon-coated copper grids (Ted Pella, California, US). Excess solution was removed with filter paper and the sample was allowed to dry at room temperature overnight. The resulting images were analyzed using ImageJ version 1.40 software (NIH, Wayne Rasband); for size analysis, at least 100 nanoparticles were evaluated per sample and the statistical analysis was performed with OriginPro2020 software (Northampton, US).

\subsubsection{Radiolabeling}

Radiolabeling was performed as previously reported [13]. Briefly, $0.5 \mathrm{~mL}$ of ${ }^{99 \mathrm{~m}} \mathrm{Tc}$ pertechnetate $(1 \mathrm{mCi})$ with $60 \mu \mathrm{L}$ of $\mathrm{SnCl} 2(10 \mathrm{mM}, \mathrm{pH} 1)$ and $2 \mu \mathrm{L}$ of $\mathrm{HCl}(12 \mathrm{M})$ were incubated at $30^{\circ} \mathrm{C}$ under constant stirring (500 rpm, $15 \mathrm{~min})$. Then, the $\mathrm{pH}$ was adjusted to $7(\mathrm{NaOH}, 1 \mathrm{M})$ for further adding $40 \mu \mathrm{L}$ of tricine $(30 \mathrm{mM}, 0.1 \mathrm{M}$ phosphate buffer, $\mathrm{pH} 7.4)$ at $30{ }^{\circ} \mathrm{C}$ under constant stirring $(500 \mathrm{rpm}$, $10 \mathrm{~min})$. Finally, $1 \mathrm{~mL}$ of each HYNIC-AuNPs-MAN $(\mathrm{h})(1 \mathrm{nM})$ and $10 \mu \mathrm{L}$ of EDDA $(20 \mathrm{mM}, 0.1 \mathrm{M}$ phosphate buffer, $\mathrm{pH} 7.4$ ) were added to the previous solution and incubated for $20 \mathrm{~min}$ at $100{ }^{\circ} \mathrm{C}$ under stirring at $500 \mathrm{rpm}$. Purification was performed by filtration using Amicon centrifugal filters $(0.5 \mathrm{~mL}$ ultracel, $100 \mathrm{~K})$ at 12,000 rpm, for $5 \mathrm{~min}$. Radiolabeled nanoparticles $\left({ }^{99 \mathrm{~m}} \mathrm{Tc}-\mathrm{AuNPs}-\mathrm{MAN}_{(\mathrm{h})}\right)$ remained in the filter, while free pertechnetate $\left({ }^{99} \mathrm{~m}_{\mathrm{TcO}_{4}}{ }^{-}\right)$and hydrolyzed/reduced technetium $\left({ }^{99 \mathrm{~m}} \mathrm{TcO}_{2}\right)$ passed through the filter. The radiolabeling efficiency was evaluated by measuring the radioactivity $(\mathrm{mCi})$ in the supernatant and pellet, using a dose calibrator(34-056 Deluxe Isotope Calibrator II, Nuclear Associates).

The radiochemical purity $(R P)$ was determined by ITLC-SG (General Electric, Santa Clara, CA, USA). The samples $(2 \mu \mathrm{L})$ were spotted on ITLC strips and 2-butanone and saline solution $(\mathrm{NaCl}-0.9 \%)$ were used as mobile phases. The 2-butanone was used to evaluate the percentage of free ${ }^{99 \mathrm{~m}} \mathrm{TcO}_{4}{ }^{-}$ 
and the saline solution was employed to measure the percentage of hydrolyzed/reduced technetium $\left({ }^{99} \mathrm{TcO}_{2}\right)$. The strips were cut in half and the radioactivity in each segment was measured using a well-type gamma counter (Ludlum 2200, Sweetwater, TX, USA). The \% RP was calculated as

$$
\% R P=\frac{B}{B+T} \times 100
$$

where $B$ and $T$ represent the radioactivity (cps) measured at the bottom (i.e., ${ }^{99 m}$ Tc-AuNPs-MAN) and top segments (i.e., ${ }^{99} \mathrm{TcO}_{4}{ }^{-}$or ${ }^{99} \mathrm{mcO}_{2}$ ), respectively.

The radiolabeling stability was measured in saline solution $(\mathrm{NaCl} 0.9 \%)$ at room temperature and in fresh human serum $\left(37^{\circ} \mathrm{C}\right)$. A total of $100 \mu \mathrm{L}$ of ${ }^{99 m} \mathrm{Tc}-\mathrm{AuNPs}-\mathrm{MAN}_{(\mathrm{h})}$ was added to $500 \mu \mathrm{L}$ of either saline solution or human serum and incubated for $6 \mathrm{~h}$. The stability, as a function of time, was determined by ITLC-SG/NaCl-0.9\%, as previously described, after collecting samples $(15 \mu \mathrm{L})$ at $15 \mathrm{~min}$; $30 \mathrm{~min}$; and 1, 2, 4, and 6h. All measurements were corrected by radioactivity decay.

\subsection{Animal Model}

Male Wistar rats (250-300 g) were obtained from the UNAM's Medical School animal facility (Mexico City). Animals were kept in a pathogen-free environment and fed with autoclaved food and water ad libitum. The procedures for care and use of the animals were approved by local institutional Scientific and Ethics Committees at INCan (019/010/IBI) (CEI/1349/18) (2019/0403/CB1) and all applicable institutional and governmental regulations were followed in accordance with the Federal Regulations for Animal Production, Care and Experimentation (NOM-062-ZOO-1999, Ministry of Agriculture, Mexico, Mexico). The guidelines from the Guide for the Care and Use of Laboratory Animals of the National Institute of Health (NIH, Washington D. C., US) were also followed. All efforts were made to minimize animal suffering and to reduce the number of animals used in the experiments.

To evaluate the potential of ${ }^{99 \mathrm{~m}} \mathrm{Tc}-\mathrm{AuNPs}-\mathrm{MAN}$ as a contrast agent for SLN mapping, the foot-draining popliteal lymph node model of rats was chosen. This model is widely used to test diagnostic techniques of metastasis and for drug delivery to lymphatic nodes. In this model, the administration of ${ }^{99} \mathrm{~m}$ Tc-AuNPs-MAN was performed in the footpad of Wistar rats. It is expected that ${ }^{99 \mathrm{~m}} \mathrm{Tc}$-AuNPs-MAN will drain from the administration area to the first lymphatic node, i.e., the popliteal lymph node.

\subsection{In Vivo SPECT/CT Imaging}

A microPET/SPECT/CT imaging system (Albira ARS, Bruker, Billerica, Spain) was used to analyze the in vivo lymphatic mapping of the radiolabeled conjugates in healthy rats. Nine rats were used in this study, organized into two experimental groups. In the first group (Group A), three rats were used to compare the popliteal node targeting of ${ }^{99} \mathrm{~m}$ Tc-AuNPs-MAN $48 \mathrm{~h}$ vs. ${ }^{99 \mathrm{~m}}$ Tc-AuNPs-ALA $48 \mathrm{~h}$. This experiment was designed to verify the lymphatic node accumulation of nanoparticles coated with mannose. The other six rats (Group B) were used to evaluate the targeting efficiency of ${ }^{99} \mathrm{~m}$ Tc-AuNPs-MAN $48 \mathrm{~h}$ vs. ${ }^{99 \mathrm{~m}}$ Tc-Sulfur colloid. This colloid is commonly used in clinical practice for the assessment of lymphatic tracking and sentinel node targeting and was used here as a gold standard.

The radiolabeled complexes were injected subcutaneously into the footpad of rats under anesthesia using a mixture of oxygen/isofluorane at 3\%. The footpad of each animal received a single injection $(\sim 50 \mu \mathrm{Ci})$ of ${ }^{99 \mathrm{~m}} \mathrm{Tc}-\mathrm{AuNPs}-\mathrm{MAN}_{48},{ }^{99 \mathrm{~m}} \mathrm{Tc}-\mathrm{AuNPs}-\mathrm{ALA}_{48 \mathrm{~h}}$, or ${ }^{99 \mathrm{~m}} \mathrm{Tc}$-Sulfur colloid in approximately $35 \mu \mathrm{L}$. After the injection, the footpad was gently massaged for one minute to promote the movement of the complex into the lymphatic pathway. For the first experiment (Group A), images were acquired at 1 and $3 \mathrm{~h}$ post injection and the animals were sacrificed by cervical dislocation after acquiring the $3 \mathrm{~h}$ image; popliteal lymph nodes and the injection site were dissected. The nodes were harvested, weighed, and counted for radioactivity using the well-type gamma counter (Ludlum Model 2200 Scaler/Ratemeter, Texas, US). For the second experiment (Group B), images were acquired at 1, 3, and 6 $\mathrm{h}$ post injection. 
All images were reconstructed with Albira's reconstruction software and the quantification analysis and image processing were performed with PMODE (PMODE Technologies, Ltd. Zurich, Switzerland) and Osirix MD (Pixmeo SARL), respectively.

\subsection{Statistical Analysis}

The results are expressed as the mean $\pm \mathrm{SD}$ (standard deviation). Statistical analysis was performed using one-way analysis of variance (ANOVA). Significance was assumed at $p<0.05$. All assays were performed with at least three independent triplicates.

Author Contributions: Conceptualization, L.A.M.; methodology, O.J.E.-M., A.R.-G., D.R.-H., and L.A.M.; imaging acquisition, processing, and analysis, O.J.E.-M., A.R.-G., D.R.-H., and L.A.M.; formal analysis, A.R.-G., F.F.C.-T., O.J.E.-M., and L.A.M.; writing-original draft preparation, A.R.-G.; writing—review and editing, A.R.-G., F.F.C.-T., and L.A.M. All authors have read and agreed to the published version of the manuscript.

Funding: This work was supported by the National Autonomous University of Mexico (UNAM; Grant DGAPA-IN104919) and the National Institute of Cancerology (INCan) (Grant 019/010/IBI).

Acknowledgments: Roberto De los Santos for his technical assistance with the imaging acquisition and processing.

Conflicts of Interest: No conflicts of interest exist in the submission of this manuscript.

\section{References}

1. Koo, H.; Huh, M.S.; Ryu, J.H.; Lee, D.-E.; Sun, I.-C.; Choi, K.; Kim, K.; Kwon, I.C. Nanoprobes for biomedical imaging in living systems. Nano Today 2011, 6, 204-220. [CrossRef]

2. Daniel, M.-C.; Astruc, D. Gold Nanoparticles: Assembly, Supramolecular Chemistry, Quantum-Size-Related Properties, and Applications toward Biology, Catalysis, and Nanotechnology. Chem. Rev. 2004, 104, 293-346. [CrossRef]

3. Mariani, G.; Bruselli, L.; Kuwert, T.; Kim, E.E.; Flotats, A.; Israel, O.; Dondi, M.; Watanabe, N. A review on the clinical uses of SPECT/CT. Eur. J. Nucl. Med. Mol. Imaging 2010, 37, 1959-1985. [CrossRef]

4. Ng, Q.K.T.; Olariu, C.I.; Yaffee, M.; Taelman, V.F.; Marincek, N.; Krause, T.; Meier, L.; Walter, M.A. Indium-111 labeled gold nanoparticles for in-vivo molecular targeting. Biomaterials 2014, 35, 7050-7057. [CrossRef]

5. Zhang, Y.; Zhang, Y.; Yin, L.; Xia, X.; Hu, F.; Liu, Q.; Qin, C.; Lan, X. Synthesis and Bioevaluation of Iodine-131 Directly Labeled Cyclic RGD-PEGylated Gold Nanorods for Tumor-Targeted Imaging. Contrast Media Mol. Imaging 2017, 2017, 6081724. [CrossRef]

6. Morales-Avila, E.; Ferro-Flores, G.; Ocampo-García, B.E.; De León-Rodríguez, L.M.; Santos-Cuevas, C.L.; García-Becerra, R.; Medina, L.A.; Gómez-Oliván, L. Multimeric System of 99mTc-Labeled Gold Nanoparticles Conjugated to $c[\operatorname{RGDfK}(\mathrm{C})]$ for Molecular Imaging of Tumor $\alpha(\mathrm{v}) \beta(3)$ Expression. Bioconjug. Chem. 2011, 22, 913-922. [CrossRef]

7. Black, K.C.; Akers, W.J.; Sudlow, G.; Xu, B.; Laforest, R.; Achilefu, S. Dual-radiolabeled nanoparticle SPECT probes for bioimaging. Nanoscale 2015, 7, 440-444. [CrossRef]

8. Wagner, T.; Buscombe, J.; Gnanasegaran, G.; Navalkissoor, S. SPECT/CT in sentinel node imaging. Nucl. Med. Commun. 2013, 34, 191-202. [CrossRef]

9. Marcinow, A.M.; Hall, N.; Byrum, E.; Teknos, T.N.; Old, M.O.; Agrawal, A. Use of a Novel Receptor-Targeted (CD206) Radiotracer, 99mTc-Tilmanocept, and SPECT/CT for Sentinel Lymph Node Detection in Oral Cavity Squamous Cell Carcinoma: Initial Institutional Report in an Ongoing Phase 3 Study. JAMA Otolaryngol. Head Neck Surg. 2013, 139, 895-902. [CrossRef]

10. Kim, C.; Zukotynski, K. Desirable Properties of Radiopharmaceuticals for Sentinel Node Mapping in Patients with Breast Cancer Given the Paradigm Shift in Patient Management. Clin. Nucl. Med. 2017, 42, 275-279. [CrossRef]

11. Keshtgar, M.R.S.; Ell, P.J. Sentinel lymph node detection and imaging. Eur. J. Nucl. Med. 1999, 26, 57-67. [CrossRef]

12. Maccora, D.; Dini, V.; Battocchio, C.; Fratoddi, I.; Cartoni, A.; Rotili, D.; Castagnola, M.; Faccini, R.; Bruno, I.; Scotognella, T; et al. Gold Nanoparticles and Nanorods in Nuclear Medicine: A Mini Review. Appl. Sci. 2019, 9, 3232. [CrossRef] 
13. Ocampo-García, B.E.; Ramírez, F.; Flor de, M.; Ferro-Flores, G.; De León-Rodríguez, L.M.; Santos-Cuevas, C.L.; Morales-Avila, E.; de Murphy, C.A.; Pedraza-López, M.; Medina, L.A.; et al. 99mTc-labelled gold nanoparticles capped with HYNIC-peptide/mannose for sentinel lymph node detection. Nucl. Med. Biol. 2011, 38, 1-11. [CrossRef]

14. Volkert, A.A.; Subramaniam, V.; Ivanov, M.R.; Goodman, A.M.; Haes, A.J. Salt-Mediated Self-Assembly of Thioctic Acid on Gold Nanoparticles. ACS Nano 2011, 5, 4570-4580. [CrossRef]

15. Toro-Cordova, A.; Flores-Cruz, M.; Santoyo-Salazar, J.; Carrillo-Nava, E.; Jurado, R.; Figueroa-Rodriguez, P.A.; Lopez-Sanchez, P.; Medina, L.A.; Garcia-Lopez, P. Liposomes Loaded with Cisplatin and Magnetic Nanoparticles: Physicochemical Characterization, Pharmacokinetics, and In-Vitro Efficacy. Molecules 2018, 23, 2272. [CrossRef]

16. Krimm, S.; Bandekar, J. Vibrational Spectroscopy and Conformation of Peptides, Polypeptides, and Proteins. In Advances in Protein Chemistry; Anfinsen, C.B., Edsall, J.T., Richards, F.M., Eds.; Academic Press: Cambridge, MA, USA, 1986; Volume 38, pp. 181-364.

17. Winter, A.; Engels, S.; Wawroschek, F. Sentinel lymph node surgery in prostate cancer using magnetic particles. Curr. Opin. Neurol. 2018, 28, 184-190. [CrossRef]

18. Seim, N.B.; Wright, C.L.; Agrawal, A. Contemporary use of sentinel lymph node biopsy in the head and neck. WJOHNS 2016, 2, 117-125. [CrossRef]

19. Whiteside, T.L. Head and Neck Carcinoma Immunotherapy: Facts and Hopes. Clin. Cancer Res. 2018, 24, 6-13. [CrossRef]

20. Krag, D.N.; Weaver, D.L.; Alex, J.C.; Fairbank, J.T. Surgical resection and radiolocalization of the sentinel lymph node in breast cancer using a gamma probe. Surg. Oncol. 1993, 2, 335-340. [CrossRef]

21. Jimenez, I.; Roca, M.; Vega, E.; García, M.; Benitez, A.; Bajén, M.; Martín-Comín, J. Particle sizes of colloids to be used in sentinel lymph node radiolocalization. Nucl. Med. Commun. 2008, 29, 166-172. [CrossRef]

22. Alazraki, N.P.; Eshima, D.; Eshima, L.A.; Herda, S.C.; Murray, D.R.; Vansant, J.P.; Taylor, A.T. Lymphoscintigraphy, the sentinel node concept, and the intraoperative gamma probe in melanoma, breast cancer, and other potential cancers. Semin. Nucl. Med. 1997, 27, 55-67. [CrossRef]

23. Akers, W.J.; Edwards, W.B.; Kim, C.; Xu, B.; Erpelding, T.N.; Wang, L.V.; Achilefu, S. Multimodal sentinel lymph node mapping with single-photon emission computed tomography (SPECT)/computed tomography (CT) and photoacoustic tomography. Transl. Res. 2012, 159, 175-181. [CrossRef]

24. Li, M.; Liu, C.; Gong, X.; Zheng, R.; Bai, Y.; Xing, M.; Du, X.; Liu, X.; Zeng, J.; Lin, R.; et al. Linear array-based real-time photoacoustic imaging system with a compact coaxial excitation handheld probe for noninvasive sentinel lymph node mapping. Biomed. Opt. Express BOE 2018, 9, 1408-1422. [CrossRef]

25. Azad, A.K.; Rajaram, M.V.S.; Schlesinger, L.S. Exploitation of the Macrophage Mannose Receptor (CD206) in Infectious Disease Diagnostics and Therapeutics. J. Cytol. Mol. Biol. 2014, 1, 5.

26. Rosenblum, D.; Joshi, N.; Tao, W.; Karp, J.M.; Peer, D. Progress and challenges towards targeted delivery of cancer therapeutics. Nat. Commun. 2018, 9, 1410. [CrossRef]

27. Haiss, W.; Thanh, N.T.K.; Aveyard, J.; Fernig, D.G. Determination of Size and Concentration of Gold Nanoparticles from UV-Vis Spectra. Anal. Chem. 2007, 79, 4215-4221. [CrossRef]

28. Gu, F.; Hu, C.; Tai, Z.; Yao, C.; Tian, J.; Zhang, L.; Xia, Q.; Gong, C.; Gao, Y.; Gao, S. Tumour microenvironment-responsive lipoic acid nanoparticles for targeted delivery of docetaxel to lung cancer. Sci. Rep. 2016, 6, 36281. [CrossRef]

29. Ogawa, K.; Mukai, T.; Inoue, Y.; Ono, M.; Saji, H. Development of a Novel 99mTc-Chelate-Conjugated Bisphosphonate with High Affinity for Bone as a Bone Scintigraphic Agent. J. Nucl. Med. 2006, 47, $2042-2047$.

30. Lowry, G.V.; Hill, R.J.; Harper, S.; Rawle, A.F.; Hendren, C.O.; Klaessig, F.; Nobbmann, U.; Sayre, P.; Rumble, J. Guidance to improve the scientific value of zeta-potential measurements in nanoEHS. Environ. Sci. Nano 2016, 3, 953-965. [CrossRef]

(C) 2020 by the authors. Licensee MDPI, Basel, Switzerland. This article is an open access article distributed under the terms and conditions of the Creative Commons Attribution (CC BY) license (http://creativecommons.org/licenses/by/4.0/). 